\title{
Challenges and opportunities in collaborative business process management: Overview of recent advances and introduction to the special issue
}

\author{
Chengfei Liu • Qing Li • Xiaohui Zhao
}

Published online: 21 May 2008

(C) Springer Science + Business Media, LLC 2008

\begin{abstract}
Modern business process management expands to cover the partner organisations' business processes across organisational boundaries, and thereby supports organisations to coordinate the flow of information among organisations and link their business processes. With collaborative business processes, organisations can create dynamic and flexible collaborations to synergically adapt to the changing conditions, and stay competitive in the global market. Due to its significant potential and value, collaborative business processes are now turning to be an important issue of contemporary business process management, and attracts lots of attention and efforts from both academic and industry sides. In this paper, we review the development of B2B collaboration and collaborative business processes, provide an overview of related issues in managing collaborative business processes, and discuss some emerging technologies and their relationships to collaborative business processes. Finally, we introduce the papers that are published in this special issue.
\end{abstract}

C. Liu $\cdot$ X. Zhao $(\bowtie)$

Centre for Information Technology Research,

Faculty of Information and Communication Technologies,

Swinburne University of Technology,

Melbourne, Australia

e-mail: xzhao@groupwise.swin.edu.au

C. Liu

e-mail: cliu@groupwise.swin.edu.au

Q. Li

Department of Computer Science, City University of Hong Kong,

Tat Chee Avenue,

Kowloon, Hong Kong, China

e-mail: itqli@cityu.edu.hk
Keywords Collaborative business process $\cdot$ Business process management $\cdot$ Service-oriented architecture . Process change and evolvement $\cdot$ Pervasive computing

\section{Introduction}

With the booming business globalisation and commoditisation, enterprises tend to be more dependent on business process management (BPM) to keep their business processes in control (Smith and Fingar 2003). By Gartner Inc., BPM has been ranked as the third of the top 10 strategic technologies for 2008, after green IT and unified communication (http://www.gartner.com/). As predicated by IDC, Australia, the revenue for BPM market will climb up from US\$ 1.065 billion 2005 to US\$ 3.8 billion by 2012 (http:// www.idc.com.au).

Such globalisation trend highly urges the dynamic collaborations among organisations. The business processes of different organisations are correspondingly required to be integrated seamlessly to adapt to the continuously changing business conditions, and to stay competitive in the global market. Though current business process technologies have achieved a certain level, there is still a large room between the available supports and the requirements from real collaboration scenarios. Especially in a loosely coupled collaboration environment, many non-functional yet crucial aspects, such as privacy and security, reliability and flexibility, scalability and agility, process validation, QoS guarantees, etc., are with an outstanding lack of sufficient supports. This gap in turn poses an obstacle to further advancements and wider application of business process technologies. Therefore, more academic research, facilitating infrastructures, protocols and standards are being expected to shift current BPM for supporting collaborative business processes. 
This paper aims for a deeper understanding of the main issues in modern BPM, particularly in the area of collaborative business processes, by reviewing the history, trends and related technologies of collaborative business processes. Some open issues and research opportunities in the management of collaborative business processes are also identified on the basis of the authors' knowledge and opinions.

\section{B2B collaboration and collaborative business processes}

Nowadays, B2B collaboration comprises about $85 \%$ of eCommerce volume (Turban et al. 2005). It covers a broad spectrum of applications that enable an enterprise to form electronic relationships with its distributors, resellers, suppliers, customers, and other partners. The potential business value of streamlining inter-enterprise business processes has fuelled a renewed interest in process management technologies (Dayal et al. 2001).

To support global collaborations, business processes expand to include internal and external partners, systems, and resources. According to the market forecasts from Forrester Research (http://www.forrester.com), American eCommerce market is expected to grow from $\$ 172$ billion in 2005 to $\$ 329$ billion in 2010. Moreover, it has been envisaged that the European eCommerce market may surge to $€ 263$ billion in 2011, and the total spending for business process outsourcing will rise from $€ 11.0$ billion in 2006 to $€ 18.9$ billion in 2011 with a compound annual growth rate at $11.5 \%$. In this scenario, a business process is expected to conduct collaborative business across multiple organisations. Consequently, relevant business processes across participating organisations are integrated for efficient functioning of business in the global market. This kind of integrated business process is called collaborative business process (Chen and Hsu 2001). Inevitably, a collaborative business process will definitely confront more dynamics from the contained external business processes, and more complicated relationship between participating organisations. Therefore, for the organisations of this period, BPM is expected to offer a breakthrough that makes end-to-end, dynamic, expanding, contracting and ever changing business processes manageable.

\section{Research issues in collaborative business processes}

As organisations exert to be agile in adapting their business processes to market dynamics, organisations should look beyond the traditional organisations and marketplaces through collaborative interactions and dynamic e-business solution bindings (Leymann and Zhang 2005). In the B2B collabora- tion stage, BPM is required to provide the capability for dynamic discovery of trading partners and service providers for organisations. Moreover, BPM enables federated security mechanisms, solution monitoring and management over collaborating business processes in a loosely coupled collaboration environment. Particularly, supports are highly required for managing collaborative business processes in the following aspects (Smith and Fingar 2003):

- Describe the business services that organisations can provide or they need from partners in service level agreements.

- Harmonise the enactment of collaboration by coordinating the participating organisations according to defined business processes.

- Maximise the autonomy of organisations during collaborations, and thereby ensuring organisations to benefit most from their own business objectives.

- Represent the partnerships between collaborating organisations during collaborations, and update the partnership changes.

- Guarantee the business privacy of organisations during collaborations.

- Allow specialists and other third parties to monitor, measure, and oversee the execution of business processes.

In recent years, previous efforts target at the primitive aspects of business process collaboration management, such as universal business process specification languages, system infrastructures and communication protocols across heterogeneous platforms, such as WS-BPEL (Andrews et al. 2003), ebXML (http://ebxml.org), RosettaNet (http://www.rosettanet.org), IBM Web Sphere (IBM 2005), etc. These achievements currently provide a technical foundation for basic collaboration functionalities, such as business service description, partnership representation, etc. Yet, there is still a great demand for further advancement surrounding the collaborative business process management domain, particularly in the non-functional aspects, like privacy, autonomy, dynamics, etc. In this section, we will discuss some of the mentioned issues, while the discussion may extend to other issues due to the inter-influences.

\subsection{Modelling collaborative business processes}

Modelling is an indispensable part of BPM, as modelling bridges the requirement engineering and business automation, and initiates the lifecycle of BPM. Some business process languages, like WS-BPEL (Andrews et al. 2003) and Business Process Modelling Notation (BPMN) (OMG, BPMI 2006), have been proposed to support the modelling of collaborative business processes, and are being adopted as industry standards. In this section, we are to discuss issues on contracting between organisations, object-oriented 
modelling perspective and human aspects in modelling, in the context of collaborative business processes.

\section{- Contracting and process modelling}

Collaborative business processes are used to facilitate collaborations, while collaborations origin from contracting between organisations. Traditional BPM mainly attempts to automate business processes from a computer-oriented perspective, i.e., the approaches emphasis on the data, structural and other technical aspects of business processes. Actually, there is an outstanding difference between human-oriented contracts and computer-oriented business processes, and the interpretation of contracts affects the effectiveness of the modelled collaborative business processes. Previous work on contracting (Milosevic et al. 2006; Colombo et al. 2002; Chiu et al. 2002) have ever discussed how to help human analysts interpret user requirements and convey them to business process modelling. However, there are still a lot of open issues, such as the strategies of translating the descriptive words in contracts, the approaches for mapping the extracted information to data constraints, structural constraints, etc., the criteria on evaluating and validating the contract translation, and the reuse of previous knowledge or existing business processes when modelling new business processes, and so on.

- Object-oriented view in modelling collaborative business processes

Due to the heterogeneous nature of the application environment, the participating business processes of a collaborative business process exchange information and data in a loosely coupled mode, as most implementation approaches like WS-BPEL (Andrews et al. 2003), ebXML (http://ebxml.org), RosettaNet (http://www.rosettanet.org), IBM Web Sphere (IBM 2005), etc., enable the communication in a collaborative business process with messaging mechanisms. In this communication mode, the objectoriented methodology is more appropriate to model the behaviours between the participating business processes. The deployment of object-oriented (or artifact-oriented) perspective in collaborative business process modelling has ever been investigated (Küster et al. 2007; Liu et al. 2007; Bhattacharya et al. 2007), and the object-oriented perspective shows the advantages in representing the data flows inside or between business processes.

\section{- Concerns on human aspects}

Human activities are considerably involved in most business processes, as business processes are originally developed to help people conduct businesses. However, recent efforts in BPM focus on enhancing the automation level of business processes, and therefore nowadays business process models become more computer-friendly, yet not that people-friendly. Integrating human activities to BPM will definitely better the controllability of business processes, and improve the practical performance of business processes. Some conceptual level and technical level initiatives towards this issue include process-aware information systems (Dumas et al. 2005), BPEL4People (IBM 2007), etc.

\subsection{Tracking collaborative business processes}

A collaborative business process runs across multiple organisations, and therefore tracking becomes particularly important for a participating organisation to perceive the progress of the collaboration. Yet, in the context of collaborative business processes, tracking is complicated by the cardinality of participating business processes, and the correlation of collaborating process instances. All collaborating business processes join up to be a complex network, and each business process in this network may participate in multiple collaborations with one or more process instances. In such a network, a logical instance for a collaborative business process consists of a series of process instances which collaborate together towards a common goal (Chen and Hsu 2001), and this network contains several such logical instances. Zhao and Liu (2006) have analysed the tracking network with a matrix-based model, while (Zhao et al. 2007) have investigated the workflow cardinality and instance correlations with a Petri net based approach. These work bring some basic ideas to tackle this issue.

\subsection{Privacy and confidentiality}

Privacy and confidentiality of collaborative business process modelling and enactment are of particular importance in the business collaboration scenario, where trust and security issues are highly featured. Traditional inter-organisational business process approaches present the same process for all participating organisations, and therefore neutralises the diversity of participants in terms of authority levels and perception scopes. To accommodate such diversity and protect the business privacy of organisations, a concept of process view has been proposed to vary the perceived views of different organisations towards the same collaborative business process.

van der Aalst and Weske (2001) have proposed a "topdown" workflow modelling scheme in their public-to-private approach. Later, Liu and Shen (2001), Chiu et al. (2004), Shan et al. (2005) contributed to the algorithm for constructing process views from given workflows, and the deployment of workflow views in the inter-organisational collaboration environment, respectively. Schulz and Orlowska (2004) applied another workflow view approach to provide multi-granular privacy control for the communi- 
cation between involved entities. Zhao et al. (2005) established a relative workflow model, which allows describing a collaborative business process from the perspective of individual organisations. Perrin and Godart (2004) presented an approach for process management and coordination based on synchronisation points between process services, where the personalisation on internal processes is allowed. Issam et al. (2006) proposed another view-based approach to support inter-organisational workflow cooperation, which treats an inter-organisational workflow as the cooperation of several pre-established workflows of different organisations. Some standard institutions also start adopting the concept of process view, for example, the latest business process definition meta model (BPDM) (OMG 2003) specification from OMG has defined abstract processes, collaboration processes, and private processes to represent a collaborative business process. Some initial work also attempted to incorporate process views into WS-BPEL (Zhao et al., submitted to BPM '08).

Although the adoption of process views provides a good control on privacy and confidentiality, such benefits are obtained at the cost of complexity in process representation, loads of view updating, etc. All these issues put challenges to the modelling, execution and monitoring of collaborative business processes, yet also open the following research opportunities:

\section{- Perception analysis between entities}

The essence of process views is based on the perception authority between collaborating entities. Currently, most process view approaches assume that the constraints on process visibility are pre-defined by users. Zhao et al. (2005) attempted to extract such visibility information by decomposing and analysing the content of contracts. To automate the process of defining visibility constraints, analysis on contracts or service level agreements are needed.

\section{- Process view coordination}

A process view only represents a partial view of the actual collaborative business process, and such partial views inevitably result in the variability of business process representations between different views. Such variability complicates the collaboration coordination and execution across organisational boundaries. Consequently, there require precise coordination mechanisms to maintain the correlations between process views and business processes, process views and process instances, and thereby ensure the collaboration execution in a process view environment.

\section{- Deployment of process views}

Some complex collaboration systems, like business ecosystems, virtual organisation alliances, etc., are featured by the dynamic and complex relationships between the member organisations. Most process view approaches create views in a bilateral mode, i.e., an organisation creates a process view for each partner organisation. Yet, this bilateral mode easily results in increasing loads of view updating in a changing environment. To better sort out the perception relationships and improve the efficiency of process views, comprehensive view models are on demand to systematically describe and analyse the relationship between process views and perceptive entities.

Beyond B2B collaborations, process views are also applicable to some B2C collaborations, like tourism management and customer relationship management, which are featured with the high customisability and personalisation. In such scenarios, although different customers have different requests, the service provider only runs one business process to serve all these requests. Therefore, at the customer's side, each customer views a personalised collaboration process; yet at the service provider's side, only one business process is running to serve all these customers.

\subsection{Process evolvement management}

Smith and Fingar have commented current varying market opportunities that "Changes has become the only certainty." (2003) in nowadays business globalisation background. To stay efficient and effective in such a turbulent environment, organisations are required to adapt their structures and business processes to new conditions continuously (van der Aalst et al. 2003; Khoshafian 2006). As a response, organisations are seeking new facilitating technologies to manage their dynamic, expanding and changing business processes (Kock 2006; Liu et al. 1998).

Initial efforts towards process evolvement include workflow evolution and adaptive workflows. Casati et al. (1998) presented a workflow modification language (WFML) to support modifications of a workflow model, and also discussed the case evolution policies and devised three main policies to manage case evolution. Hamadi and Benatallah (2004) proposed a self-adaptive recovery net (SARN) to support workflow adaptability during unexpected failures. Project $A D E P T_{\text {flex }}$ conducted extensive studies on schema evolution in process management, covering common workflow type and instances changes, as well as disjoint and overlapping process changes (Reichert and Dadam 1998; Rinderle et al. 2004).

On the other hand, the competence of an organisation is also subject to the compliance towards the compound of business rules, polices, processes and systems (Sadiq et al. 2007; Morgan 2002; Debevoise 2005). To consider all these factors under one umbrella, a new concept of service continuity is now proposed to architect the process evolvement and service resilience from a holistic perspective (Zhao 2008). In the pursuit of service continuity, we 
identify the following research opportunities related to process evolvement:

\section{- Rule-based process transformation}

To guarantee the process evolvements are compliant to business policies and regulations, it is necessary to computerise the real world business rules, and integrate them to BPM. Some initial work (Lang et al. 1997) modelled business rules with event-condition-action structures, or used rule interceptor services to integrate rules to some process modelling languages (Rosenberg et al. 2006). Some industry standards are also established dedicatedly for business rules, such as SBVR (OMG 2006) and PRR (OMG 2007), intending to uniform the representation of business rules.

\section{- Coordination of launching changes}

Though a quick fix to a rule may intuitively help an organisation accommodate to a change of a business process immediately, yet it may cause serious side effects in logical consistency and system stability. Especially in a large scale organisation, such casual rule editing from different departments may easily lead to chaos. Therefore, changes have to be introduced in an organised way. The potential conflicts, counteractions of rules and their influences to business processes should be pre-analysed, and thereafter these changes are to be grouped and launched according to the analysis at proper time points, for the purpose of maintaining the sanity of the system.

\section{- Process continuity and capacity management}

Process continuity refers to the continuous operation of business processes. From the business process perspective, this issue requires the structural analysis and optimisation, the flexible role assignment and resource allocation, and alternative processes for potential disruptions, etc. Capacity management is connected with maintaining a certain level of service and performance against the background of continual changes. This management denotes the on-going process of maintaining the operation continuity with reliable service availability, under the pressure of limited system capacity and demand thrusts.

\section{- Version management of process evolvement}

Versions are used to track and trace the process evolvement, and help control the process evolvement. In the process evolvement context, an instance of a business process may undergo multiple versions during its life cycle. Further, a business process may own multiple evolvement options to adapt to temporary changes or alternations. Therefore, at a moment of time, multiple process instances of different versions may exist, and such complex evolvement cases can hardly be identified by simple linear versioning methods.
Zhao and Liu (2007) have proposed a version preserving graph model to track the complex process evolvements.

\section{Facilitating technologies}

With recent IT development, some technological and methodological advances turn out to synergise current BPM with profound influences and potentials (Khoshafian 2006). Here, we briefly introduce the two most typical technologies, i.e., service-oriented architecture (SOA) and pervasive computing.

- SOA and semantic services provide a platform for distributed computing and establish a new computing paradigm that can benefit collaborations.

- Pervasive computing enables the communication to individual objects, and thereby provides a supporting environment for real time BPM in a geography distributed environment.

In addition, there are also other technologies leading to the evolvement of BPM. Understanding these new technology implications and the new applications is a key to enable researchers and practitioners to face these new challenges to the domain of collaborative business processes.

\subsection{Service-oriented architecture}

As a new cross-discipline paradigm, SOA addresses how to enable IT and computing technology to help people perform business processes, services, and applications more efficiently and effectively, ultimately resulting in a gain to the organisation. SOA currently shapes the processes of business modelling, solution creation, service delivery, and software architecture design, development and deployment. Within this trend, issues such as service composition, service discovery, service monitoring, service orchestration, etc., emerge out during the adoption of SOA.

The paradigm shift towards SOA calls for technological and methodological advances, and here we identify the following research issues and corresponding opportunities from the perspective of collaborative business processes:

- Incorporating the service network into the business network

Current service network is constructed by the functional software components with specific interfaces, which do not necessarily correspond to business services. To seamlessly combine such software services and business services, the services are required to be well characterised, located and managed according to their business properties in an intelligent and dynamic manner. Technically, this requirement places challenges to semantic service modelling, 
quality/policy-based service discovery/selection through direct and compositional matching, etc. Ontology based semantic services are considered as the promising methods to achieve this goal.

\section{- Running business processes over the service network}

Specifications, like WS-BPEL, WS-CDL (Kavantzas et al. 2004), etc., are established to support the service orchestration and choreography according to pre-defined processes or rules. Yet, currently these specifications mainly define the processes and related rules from the technical perspective, i.e., focus on the input/output parameters, message correlations, etc. Such a static coordination mode is hard to capture the dynamics of business processes in collaborations. Supports on dynamic and flexible creation of business processes, service composition and aggregation, service governance, etc., are on demand to facilitate the business process execution over the service network.

- Reliability and consistency of service operations and transactions

Due to the long-duration and loosely coupled properties, Web service based applications require more transactional supports beyond traditional transactions. Some initial work has been done to deal with the transaction aspect of Web service applications, in form of standards or protocols. Among them are BPEL4WS, WS-Coordination (Cabrera et al. 2005), WSTransaction (Cox et al. 2004), WSCI (Arkin et al. 2002), and WS-CDL. Compensation is the basic mechanism adopted in all of these standards for backward recovery. However, the compensation mechanism adopted is too fixed and cannot satisfy the various requirements of different applications. Liu and Zhao (2008) have investigated on improving the flexibility of compensations to accommodate the diversity of operation failures, by extending WSDL and WS-BA with a flexible compensation mechanism which enables the multiple choices of compensations for each Web service operation. In case that the compensating task for a task does not exist, Liu et al. (2001) have introduced the notion of confirmation to cope with this problem. Flexible lightweight transaction management is longed for to enhance the reliability and consistency for business collaborations in service oriented architecture.

\subsection{Pervasive computing and real time process management}

A world in which virtually every object has processing power with wireless or wired connections to a global network is the world of pervasive computing (Turban et al. 2005). The term pervasive computing also goes by the names ubiquitous computing or augmented computing. Such technologies create tremendous opportunities for linking various objects from real worlds, and therefore provide an ideal platform for real time BPM.
Radio frequency identification (RFID) is a typical wireless communication technology to support pervasive computing. In recent years, RFID has gained a significant momentum and is emerging as an important technology for revolutionising a wide range of applications including supply chain management, retail, aircraft maintenance, baggage handling, healthcare, etc. (EII 2007; Glover and Bhatt 2006; Götz et al. 2006). A bright forecast from IDTechEx expects that the total RFID market value (including all hardware, systems, integrations etc.) across all countries will rise from $\$ 4.96$ billion in 2007 to $\$ 26.88$ billion in 2017 (http://www.idtechex.com).

Currently, enterprises apply RFID mainly to facilitate the tracking and tracing of material flows, particularly in collaboration environments. Due to the association between material flows and business processes, there are great potentials between BPM and material flow management. Thus, the further advancement and deployment of RFID undoubtedly urge the combining BPM with RFID applications.

Managing the business processes and material flows with the large and complex RFID Data and their underlying dynamically configured networks is a challenging research topic. More specifically we can identify the following research opportunities:

\section{- Formalisation of business processes and RFID data}

A critical issue for both RFID data processing and management is on how to define a computational model that can process and manage RFID data in an evolving environment where the configuration of RFID readers is dynamic, and the RFID tagged objects are constantly moving. Recently, Gonzalez et al. (2006) and Han et al. (2006) proposed a probabilistic workflow approach to describe the probability of the tagged objects transit between locations. Although, this workflow model is mainly developed for data mining purpose, this work already indicated the commencement of incorporating BPM into RFID applications.

\section{- Swarming behaviours}

In the transportation stage, many RFID items act in the similar behaviour, and follow the same path. For example, all the parts packed in a box follow the same movement behaviour during the transportation, and these parts may be dispatched to different places in groups when they arrive to the distribution centre. A good modelling and utilisation of this phenomenon can greatly improve the tracking efficiency, and in turn enhance the performance of business processes.

\section{- RFID network dynamics}

Real world RFID networks are always changing over time. How the dynamic configuration of RFID networks of their readers in topology and connections influences the 
upper layer business processes? And how BPM systems pro-actively re-arrange the RFID networks to better accommodate the collaborations, and further realise a kind of business process driven self-organising network? These are all open issues for the incorporation of BPM into RFID applications.

\section{Introduction to the papers in this special issue}

This special issue consists of seven quality papers that we selected from 40 submissions after a serious review process. These papers present the latest research on collaborative business processes in aspects of process modelling, QoS management, transaction management, privacy and security, and implementation techniques. We are very grateful to the efforts of all authors and reviewers.

Paper "Analysis of a Collaborative Workflow Process with Distributed Actors" has presented an empirical study on the evaluation of an industrial workflow process where similar tasks can be performed by various actors at many different locations.

Paper "Analysis of Composed Services through Fault Injection" has addressed the quality assessment of composed services in terms of fault tolerance. Two aspects are particularly discussed, i.e., the effect of delays on complex services and the reactions of composed services to data faults.

Paper "Delegating Revocations and Authorizations in Collaborative Business" has discussed the access control in the collaborative business environment with a role-based delegation and revocation model. Revocations are classified in terms of dependency, resilience, propagation and dominance.

Paper "CoBTx-Net: A Model for Business Collaboration Reliability Verification" has presented a chorographical business transaction net (CoBTx-Net) to specify collaborative business transaction and manage the collaboration by individual participants.

Paper "Evaluation of Inter-Organizational Business Process Solutions: a Conceptual Model-based Approach" has proposed to use Generic Process Model framework and Bunge's ontology to conceptually capture inter-organisational business processes. The approach is claimed to be generic, and can evaluate different implementation approaches for inter-organisational business processes, such as P2P, RosettaNet and CWM, which are tested in the paper for the evaluation in aspects of privacy and autonomy.

Paper "Towards Ubiquitous Tourist Service Coordination and Process Integration: a Collaborative Travel Agent System with Semantic Web Services" has introduced a collaborative travel agent system (CTAS) and a multi-agent information system for CTAS based on a case study of a large service-oriented travel agency. Authors have also shown how the ontology from semantic Web services help tourists better plan, understand and specify their requirements collaboratively with CTAS.

Paper "Evie-A Developers Toolkit for Encoding Service Interaction Patterns" has incorporated service interaction patterns into java based event handling mechanism, and presented an approach for rapid design and deployment of event-driven collaborative business processes by extending Java language.

\section{Conclusions}

In this paper, we examined the recent advances in related areas surrounding the field of "collaborative business processes". We showed that collaborative business processes are becoming a focal issue in the continued development of B2B collaborations. We also identified some urgent research issues related to collaborative business process management, and discussed the relationship between collaborative business processes and recent technologies.

We hope that the seven articles included in this special issue will give readers a sense about the research trends in collaborative business process management, and will stimulate others to do more research in this important and promising domain. We would like to thank all the reviewers who have contributed to the review process of this special issue. Without their generous help, this special issue would not have been so successful.

\section{References}

Andrews, T., Curbera, F., Dholakia, H., et al. (2003). Business process execution language for web services (BPEL4WS) 1.1.

Arkin, A., Askary, S., Fordin, S. et al. (2002). Web service choreography interface (WSCI) 1.0.

Bhattacharya, K., Gerede, C. E., Hull, R., Liu, R., \& Su, J. (2007). Towards formal analysis of artifact-centric business process models. In Proceedings of the 5th International Conference on Business Process Management 288-304

Cabrera, L. F., Copeland, G., Feingold, M., et al. (2005). Web services coordination (WS-Coordination).

Casati, F., Ceri, S., Pernici, B., \& Pozzi, G. (1998). Workflow evolution. Data \& Knowledge Engineering, 24, 211-238.

Chen, Q., \& Hsu, M. (2001). Inter-enterprise collaborative business process management. In Proceedings of International Conference on Data Engineering (pp 253-260).

Chiu, D. K. W., Cheung, S. C., Till, S., Karlapalem, K., Li, Q., \& Kafeza, E. (2004). Workflow view driven cross-organizational interoperability in a web service environment. Information Technology and Management, 5, 221-250.

Chiu, D. K. W., Karlapalem, K., Li, Q., \& Kafeza, E. (2002). Workflow view based e-contracts in a cross-organizational e-services environment. Distributed and Parallel Databases, 12(2-3), $193-216$. 
Colombo, E., Francalanci, C., \& Pernici, B. (2002) Modeling coordination and control in cross-organizational workflows. In Proceedings of DOA/CoopIS/ODBASE, 91-106

Cox, W., Cabrera, F., Copeland, G. et al. (2004). Web services transaction (WS-Transaction).

Dayal, U., Hsu, M., \& Ladin, R. (2001). Business process coordination: state of the art, trends, and open issues. In Proceedings of 27th International Conference on Very Large Database, 3-13

Debevoise, T. (2005). Business process management with a business rules approach. Business Knowledge Archetects.

Dumas, M., van der Aalst, W. M. P., \& ter Hofstede, A. H. M. (2005). Process-aware information systems. Wiley

EII (2007) RFID data processing \& management.

Glover, B., \& Bhatt, H. (2006). RFID essentials. O'Reilly

Gonzalez, H., Han, J., \& Li, X. (2006). Mining compressed commodity workflows from massive RFID data sets. In Proceedings of the 15th International Conference on Information and Knowledge Management (pp. 162-171).

Götz, T., Safai, S., \& ,Beer, P. (2006). Efficient supply chain management with SAP solutions for RFID. Galileo Press

Hamadi, R., \& Benatallah, B. (2004). Recovery nets: Towards selfadaptive workflow systems. In Proceedings of the 5th International Conference on Web Information Systems Engineering (pp. 439-453).

Han, J., Gonzalez, H., Li, X., \& Klabjan, D. (2006). Warehousing and mining massive RFID data sets. In Proceedings of the 2 nd International Conference on Advanced Data Mining and Applications (pp. 1-18)

IBM (2005). IBM websphere business integration handbook.

IBM (2007). WS-BPEL extension for people. http://www.ibm.com/ developerworks/webservices/library/specification/ws-bpel4people/.

Issam, C., Schahram, D., \& Samir, T. (2006). The view-based approach to dynamic inter-organizational workflow cooperation. Data \& Knowledge Engineering, 56, 139-173.

Kavantzas, N., Burdett, D., \& Ritzinger, G. (2004). Web services choreography description language (WS-CDL) 1.0.

Khoshafian, S. (2006). Service oriented enterprise. Auerbach Publisher.

Kock, N. (2006). System analysis \& design fundamentals - A business process redesign approach. Sage Publications.

Küster, J. M., Ryndinam, K., \& Gall, H. (2007). Generation of business process models for object life cycle compliance. In Proceedings of the 5th International Conference on Business Process Management, $165-181$

Lang, P., Obermair, W., \& Schrefl, M. (1997). Modeling business rules with situation/activation diagrams. In Proceedings of the 13th International Conference on Data Engineering (pp. 455-464).

Leymann, F., \& Zhang, L.-J. (2005). Journal description. International Journal of Business Process Integration and Management

Liu, C., Orlowska, M. E., \& Li, H. (1998) Automating handover in dynamic workflow environments. In Proceedings of 10th International Conference on Advanced Information Systems Engineering, $159-171$

Liu, C., Orlowska, M. E., Lin, X., \& Zhou, X. (2001). Improving backward recovery in workflow systems. In Proceedings of the 7th International Conference on Database Systems for Advanced Applications, 276-283

Liu, C., \& Zhao, X. (2008). Towards flexible compensation for business transactions in web service environment. ServiceOriented Computing and Applications.

Liu, D. -R., \& Shen, M. (2001). Modeling workflows with a processview approach. In Proceedings of the 7th International Conference on Database Systems for Advanced Applications, 260-267

Liu, R., Bhattacharya, K., \& Wu, F. Y. (2007). Modeling business contexture and behavior using business artifacts. In Proceedings of the 19th International Conference on Advanced Information Systems Engineering, 324-339.

Milosevic, Z., Sadiq, S. W., \& Orlowska, M. E. (2006). Towards a methodology for deriving contract-compliant business processes. In Proceedings of the 4th International Conference on Business Process Management, 395-400

Morgan, T. (2002). Business rules and information systems-Aligning IT with business goals. Addison-Wesley.

OMG (2003). Business process definition meta model.

OMG (2006). Semantics of business vocabulary and business rules specification.

OMG (2007). Production rule representation.

OMG, BPMI (2006). Business process modeling notation (BPMN)

Perrin, O., \& Godart, C. (2004). A model to support collaborative work in virtual enterprises. Data \& Knowledge Engineering, $50,63-86$.

Reichert, M., \& Dadam, P. (1998). ADEPTflex-Supporting dynamic changes of workflows without losing control. Journal of Intelligent Information Systems, 10, 93-129.

Rinderle, S., Reichert, M., \& Dadam, P. (2004). Disjoint and overlapping process changes: Challenges, solutions, applications. In Proceedings of 12th International Conference on Cooperative Information Systems, 101-120.

Rosenberg, F., Nagl, C., \& Dustdar, S. (2006). Applying distributed business rules-The VIDRE approach. In Proceedings of the 3rd International Conference on Services Computing (pp. 471-478).

Sadiq, S., Governatori, G., \& Namiri, K. (2007). Modeling control objectives for business process compliance. In Proceedings of the 5th International Conference on Business Process Management (pp. 149-164).

Schulz, K. A., \& Orlowska, M. E. (2004). Facilitating crossorganisational workflows with a workflow view approach. Data \& Knowledge Engineering, 51, 109-147.

Shan, Z., Chiu, D. K. W., \& Li, Q. (2005). Systematic interaction management in a workflow view based business-to-business process engine. In Proceedings of the 38th Hawaii International Conference on System Sciences

Smith, H., \& Fingar, P. (2003). Business process management-The third wave. Meghan-Kiffer Press

Turban, E., R. Kelly Rainer, J., \& Potter, R. E. (2005). Introduction to information technology. Wiley

van der Aalst, W. M. P., ter Hofstede, A. H. M., \& Weske, M. (2003). Business process management: A survey. In Proceedings of International Conference on Business Process Management, 1-12.

van der Aalst, W. M. P., \& Weske, M. (2001). The P2P approach to interorganizational workflows. In Proceedings of International Conference on Advanced Information Systems Engineering (pp. 140-156).

Zhao, X. (2008). Guaranteeing service continuity in the business process change context. Swinburne University of Technology.

Zhao, X., \& Liu, C. (2006). Tracking over collaborative business processes. In Proceedings of the 4th International Conference on Business Process Management (pp. 33-48).

Zhao, X., \& Liu, C. (2007). Version management in the business process change context. In Proceedings of the 5th International Conference on Business Process Management (pp. 198-213).

Zhao, X., Liu, C., \& Yang, Y. (2005). An organisational perspective on collaborative business processes. In Proceedings of the 3rd International Conference on Business Process Management (pp. 17-31).

Zhao, X., Liu, C., Yang, Y., \& Sadiq, W. (2007). Handling instance correspondence in inter-organisational workflows. In Proceedings of the 19th International Conference on Advanced Information Systems Engineering (pp. 51-65). 
Dr. Chengfei Liu is an associate professor at the Faculty of Information and Communication Technologies, Swinburne University of Technology, Australia. $\mathrm{He}$ is the leader of Web and Data Engineering research group and the coordinator of Service Oriented Computing research theme in the Swinburne Centre for Information Technology Research (CITR). He was a senior lecturer with the University of South Australia, a lecturer with the University of Technology Sydney, and a senior research scientist with the Cooperative Research Centre for Distributed Systems Technology in University of Queensland. He has also held visiting positions at the IBM Silicon Valley Laboratory, University of Aizu, Kyoto University, and Chinese University of Hong Kong. Dr. Liu obtained his PhD in Computer Science from the Nanjing University in 1988. His current research interests include XML and Web information systems, Web services, workflows and business process management, advanced transaction models, and data integration. He has published over 90 research papers in journals such as TODS, DKE, IJCIS, WWWJ, Information Sciences, and conferences such as VLDB, ICDE, CAiSE, BPM, ICWS and has been involved in program committees of over 50 international conferences or workshops in the areas of database systems and Web information systems.

Dr. Qing Li is a professor at the Department of Computer Science, City University of Hong Kong where he joined as a faculty member since Sept 1998. Prior to that, he has taught at the Hong Kong Polytechnic University, the Hong Kong University of Science and Technology and the Australian National University (Canberra, Australia). He has served/is serving as a consultant to Microsoft Research Asia (Beijing, China), Motorola Global Computing and Telecommunications Division (Tianjin Regional Operations Center), and the Division of Information Technology, Commonwealth Scientific and Industrial Research Organization (CSIRO) in Australia. He is a guest professor of the University of Science and Technology of China (USTC) and Zhong Shan (Sun Yat-Sen) University, a visiting professor at the Institute of Computing Technology (Knowledge Grid), Chinese Academy of Science (Beijing, China), and an adjunct professor of the Hunan University (Changsha, China) where he got his BEng. degree from the Department of Computer Science in 1982. $\mathrm{He}$ is also a guest professor (Software Technology) of the Zhejiang University (Hangzhou, China). He has been actively involved in the research community by serving as an associate editor and reviewer for technical journals, and as an organiser/co-organiser of numerous international conferences. He has served as a programme committee member for over thirty international conferences (including VLDB, ICDE, WWW, ER, CIKM, CAiSE, DASFAA, CoopIS, and FODO). $\mathrm{He}$ is currently a Senior Member of IEEE, a member of ACMSIGMOD and IEEE Technical Committee on Data Engineering. He is the chairperson of the Hong Kong Web Society, and also served/is serving as an executive committee (EXCO) member of IEEE-Hong Kong Computer Chapter and ACM Hong Kong Chapter. His research interests include object modelling, multimedia databases, and Web services.

Dr. Xiaohui Zhao is a research fellow at the Faculty of Information and Communication Technology, Swinburne University of Technology, Australia. He has obtained his $\mathrm{PhD}$ from Swinburne University of Technology in 2007, ME and BE degrees from Harbin Institute of Technology, China, in 2003 and 2001, respectively. He worked as a research assistant for National Institute of Informatics, Tokyo, Japan, from 2003 to 2004. He has published a series of peer reviewed papers in business process management area, and he has served/is serving as a PC member or reviewer for several conferences, workshops and journals. His research interests include business process management, serviceoriented architecture, and pervasive business computing. 